\title{
Assessment of Photosynthesis Tolerance to Herbicides, Heat and High Illumination by Fluorescence Imaging
}

\author{
Pedro Saura and María José Quiles*
}

Departamento de Biología Vegetal, Facultad de Biología, Universidad de Murcia, Campus de Espinardo, E-30100 Murcia, Spain

\begin{abstract}
Fluorescence imaging represents a non-invasive tool for revealing and understanding spatial heterogeneity in leaf performance caused by external factors, such as abiotic stress. Sun (Rosa meillandina and Chrysanthemum morifolium) and shade (Spathiphyllum wallisii) plants were used to study their tolerance to heat and high illumination. Fluorescence yield, effective PSII quantum yield and non-photochemical quenching were analysed in leaves attached to plants by fluorescence imaging. The control plants of all species showed homogeneous images of the fluorescence parameters throughout the leaf. The fluorescence yield (F) was 0.1 or less, the effective PSII quantum yield (Y(II)) around 0.75 and non-photochemical quenching (NPQ) less than 0.3. The two sun plants showed higher tolerance to stress conditions. Few variations were observed in $\mathrm{F}$ and $\mathrm{Y}$ (II) images after stress photoperiods and some leaf regions showed an increase in NPQ, indicating more thermal energy dissipation in these zones than in other leaf regions. The images of the fluorescence parameters were similar to those of control plants after one recovery photoperiod without stress conditions. Shade plant showed lower tolerance and irreversible damage was observed after the first photoperiod, particularly at the base of the leaf and in the areas adjacent to the ribs. The centre and top of the leaf were less damaged, and effective PSII quantum yield remained high because the leaf curved to reduce the incident radiation. Incubation with the herbicides DCMU and paraquat led to differences in the fluorescence parameter images. The effect of DCMU $(0.1 \mathrm{mM})$ was visible after 30 min incubation, beginning at the ribs and adjacent areas of the leaf. The three species studied showed different degree of sensitivity to paraquat $(0.2 \mathrm{mM})$, and the effective quantum yield in each species was affected at different incubation times.
\end{abstract}

Key Words: Chrysanthemum morifolium; DCMU; paraquat; Rosa meillandina; shade plant; Spathiphyllum wallisii; sun plant.

\section{INTRODUCTION}

The photosynthetic apparatus absorbs light energy and processes it into chemical energy. Absorption of photons excites pigment molecules and excitation energy is used in the photochemical reactions of photosynthesis. However, part of the excitation energy is dissipated by fluorescence (emission of photons by chlorophyll molecules) and heat emission, principally in the antenna system. Although photochemistry, fluorescence, and thermal energy dissipation compete in dissipating excitation energy the total energy dissipated is the sum of all three processes. Estimation of these processes under different conditions allows comparing the competition that exists among the three processes [1]. Chlorophyll fluorometry is well established as a convenient, non-invasive, rapid and quantitative technique for the investigation of photosynthesis in plants, that enables variations in the same attached leaf to be studied (for recent reviews see $[2,3])$. Based on pulse amplitude modulation (PAM) and the saturation pulse method [4], chlorophyll fluorometry provides quantitative information concerning fluorescence yield, the effective PSII quantum yield or photochemical efficiency and the non-photochemical quenching of fluorescence, which represents heat dissipation in the antenna system [5]. Three major components of non-photochemical quenching

*Address correspondence to this author at the Departamento de Biología Vegetal, Universidad de Murcia, Campus de Espinardo, E-30100 Murcia, Spain; Tel: 34968 364947; Fax: 34968 363963; E-mail: mjquiles@um.es have been identified in plants, namely, energy-dependent quenching, photoinhibitory quenching and state-transition quenching, which are related to trans-thylakoid proton gradient, photoinhibition and energy redistribution, respectively [6-8]. In recent years, the versatility of chlorophyll fluorometry has increased significantly with the development of fluorescence imaging systems which provides a powerful tool for investigating leaf photosynthesis under diverse conditions [3, $9,10]$. Fluorescence imaging reveals a wide range of internal leaf characteristics, including spatial variations due to differences in physiology and development, but may also represent a simple and effective tool for the early detection of effects caused by adverse factors [10]. Many factors, such as abiotic stress and herbicides, affect photosynthesis, causing an imbalance of excitation energy dissipation. Fluorescence imaging allows us to compare variations in energy dissipation processes and to study damage in the same attached leaf. Plants are frequently exposed to environmental stress both under natural and agricultural conditions, and it is common for more than one abiotic stress such as heat and high illumination to occur at a given time. Plants exhibit great variations in their tolerance to stress. Some plants show sufficient developmental plasticity to respond to a range of light regimes, growing as sun plants in sunny areas or as shade plants in shady habitats. However, other species of plants are adapted to either a sun- or a shade-environment, and they show different levels of tolerance to high illumination. Generally, sun plants support better exposure to high light than shade plants, which experience photoinhibition [11-14]. 
Moreover, the use of herbicides to eradicate unwanted plants is widespread in agriculture. Some herbicides, such as dichlorophenyldimethylurea (DCMU) and paraquat, block photosynthetic electron flow. DCMU acts by blocking electron flow at the quinone acceptors of PS II, by competing for the binding site of plastoquinone that is normally occupied by Qв $[15,16]$. Paraquat acts by causing oxidative stress, since this herbicide is univalently reduced by PSI to its cation radical, which rapidly donates electrons to oxygen, producing superoxide radicals $\left(\mathrm{O}_{2}^{-}\right)$[17-19]. Such superoxide production at PS I exceeds the antioxidant ability of the superoxide dismutase-ascorbate-peroxidase system and the excess superoxide and other reactive oxygen species propagate oxidative damage to other membrane components, including PS II [20]. In the present paper, we used fluorescence imaging to study the photosynthesis tolerance to heat and high illumination and the effects of the herbicides, DCMU and paraquat, in sun and shade plants. The paper presents novel images of the fluorescence parameters, which reflect the three processes of excitation energy dissipation that take place during photosynthesis.

\section{MATERIALS AND METHODOLOGY}

\section{Plant Material and Incubation Conditions}

Rosa meillandina and Chrysanthemum morifolium (sun species) and Spathiphyllum wallisii (shade species) were grown in pots at $22-25^{\circ} \mathrm{C}$ in the greenhouse under natural light conditions until flowering (control conditions). For stress conditions, adult plants were transferred to cultivation chambers under controlled watering to avoid drought stress and with $18 \mathrm{~h}$ photoperiods of high light intensity (1060 $\mu \mathrm{mol} \cdot \mathrm{m}^{-2} \cdot \mathrm{s}^{-1}$ PPFD) supplied by a $100 \mathrm{~W}$ Flood Osram white light lamp, at $35^{\circ} \mathrm{C}$ and night-periods at $24{ }^{\circ} \mathrm{C}$. For recovery after stress photoperiods, plants were exposed to $18 \mathrm{~h}$ pho toperiods of low light intensity $\left(30 \mu \mathrm{mol} \cdot \mathrm{m}^{-2} \cdot \mathrm{s}^{-1}\right.$ PPFD) sup plied by $40 \mathrm{~W} / 10$ Osram daylight fluorescent tubes, at $24{ }^{\circ} \mathrm{C}$ The experiment was duplicated for each species and in eacl plant six leaves were selected for chlorophyll fluorescencr measurements. For herbicide treatments, leaves were de tached from control plants and incubated in Petri dishes con taining water or inhibitors under white light of low intensit: (30 $\left.\mu \mathrm{mol} \cdot \mathrm{m}^{-2} \cdot \mathrm{s}^{-1} \mathrm{PPFD}\right)$ supplied by $40 \mathrm{~W} / 10$ Osram dayligh fluorescent tubes, at $24^{\circ} \mathrm{C}$.

\section{Chlorophyll Fluorescence Measurements}

Chlorophyll fluorescence was imaged, using the MINI version of the Imaging- PAM (Heinz Walz GmbH, Ef feltrich, Germany), in selected leaves attached to plant grown under control and stress conditions, or in detacher leaves in the case of herbicide treatments. Measurement: were made after the last night period in attached leaves $o$ after $30 \mathrm{~min}$ darkness in detached leaves. The fluoromete used employs the same blue LEDs for the pulse modulater measuring light, continuous actinic illumination and satura tion pulses. The minimal fluorescence yield $\left(\mathrm{F}_{0}\right)$, and the maximal fluorescence yield $(\mathrm{Fm})$ were measured in darkadapted samples. Fo was measured at low frequency of pulse modulated measuring light, while Fm was measured using a saturation pulse. This was followed by $2 \mathrm{~min}$ exposure to 50 $\mu \mathrm{mol} \cdot \mathrm{m}^{-2} \cdot \mathrm{s}^{-1} \mathrm{PAR}$, with measurements of $\mathrm{F}$, the fluorescence yield, and F'm, the maximal fluorescence yield in illuminated samples. Images of the effective PS II quantum yield of illuminated samples; Y(II), and non-photochemical quenching (NPQ), were automatically calculated by the ImagingWin software from the equations: $\mathrm{Y}(\mathrm{II})=\left(\mathrm{Fm}{ }^{\prime}-\mathrm{F}\right) / \mathrm{Fm}$ ' and $\mathrm{NPQ}=$ (Fm-Fm')/Fm'. Results are shown as color-coded images of $\mathrm{F}, \mathrm{Y}(\mathrm{II})$, and NPQ.

\section{RESULTS}

\section{Effect of Heat and High Illumination}

The fluorescence imaging technique was used to examine the inhibition of photosynthesis in intact leaves attached to plants after exposure to photoperiods with high illumination and heat. Figs. (1 and 2) show images of F, Y(II) and NPQ from a typical leaf of $C$. morifolium (Fig. 1) and R. meillandina (Fig. 2). For purposes of comparison, data from the analyzed entire leaves were also averaged and the medium values are shown in the histograms. Leaves from control plants showed images with a homogeneous colour throughout
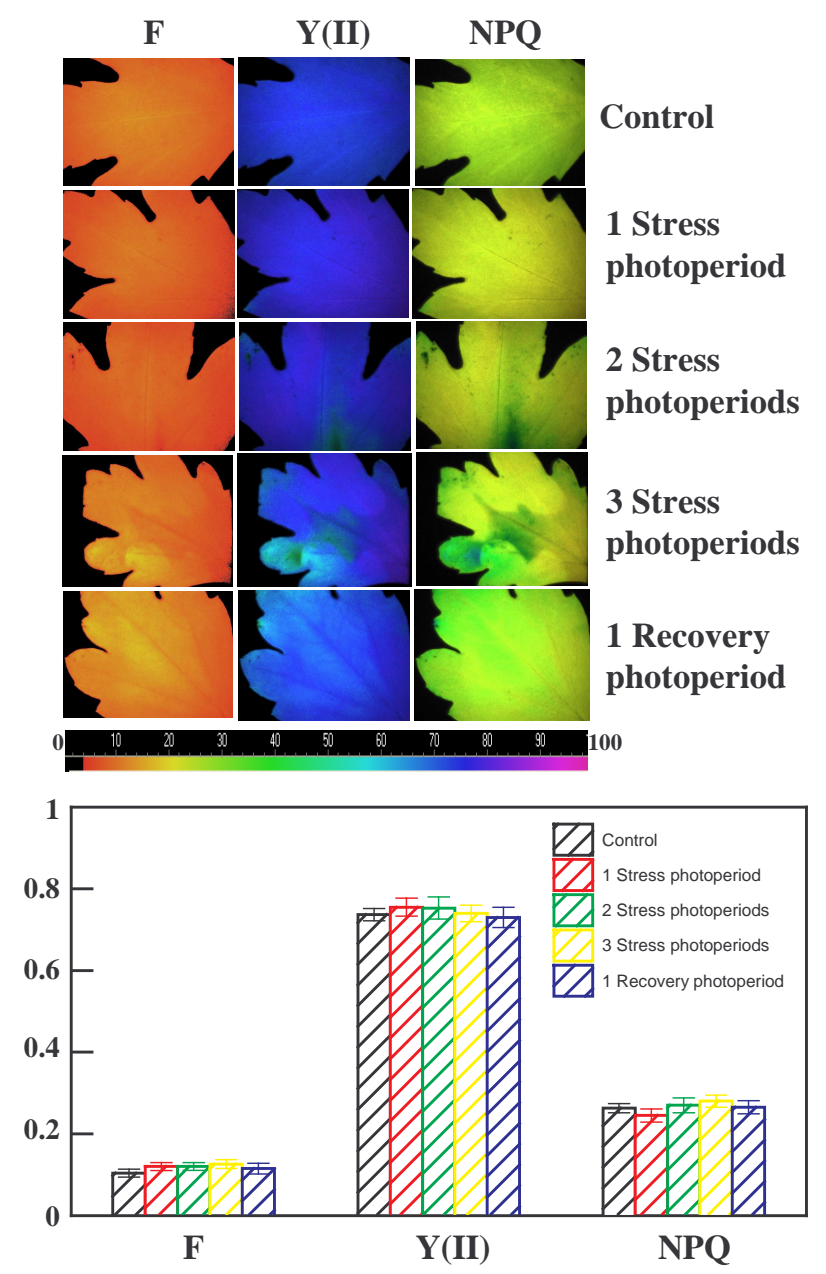

Fig. (1). Images of (F), (Y(II)), and (NPQ) from a typical leaf attached to $C$. morifolium plant, in control conditions, exposed to stress photoperiods $\left(18 \mathrm{~h}, 1060 \mu \mathrm{mol} . \mathrm{m}^{-2} \cdot \mathrm{s}^{-1} \mathrm{PPFD}\right.$ and $\left.35^{\circ} \mathrm{C}\right)$ and after one recovery photoperiod $\left(18 \mathrm{~h}, 30 \mu \mathrm{mol} \cdot \mathrm{m}^{-2} \cdot \mathrm{s}^{-1} \mathrm{PPFD}\right.$ and 24 $\left.{ }^{\circ} \mathrm{C}\right)$. Images are color coded according to the pattern ( 0 to $1 \times 100$ range) shown below the images. The histograms show the means \pm SE of parameters calculated from variable chlorophyll fluorescence measurements in six entire leaves. 

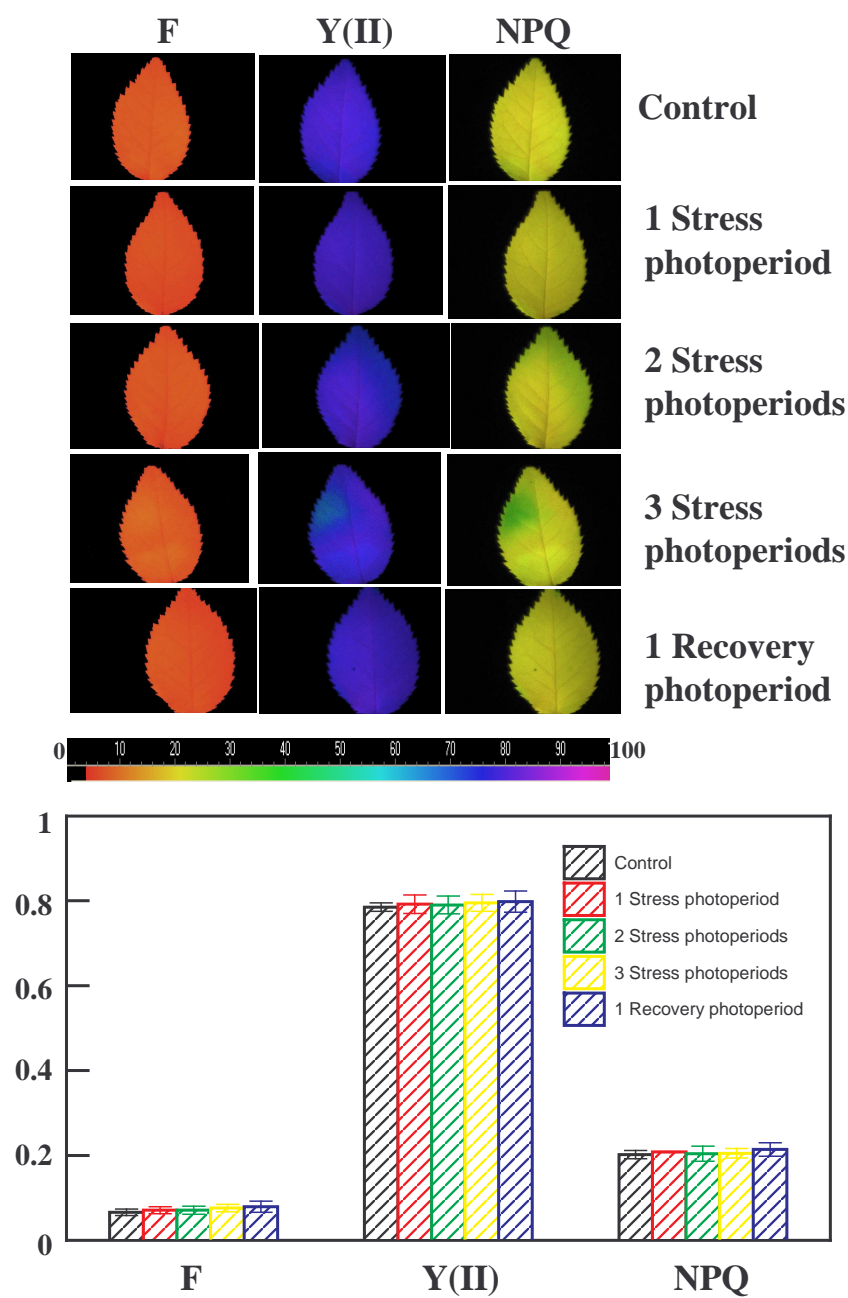

Fig. (2). Images of (F), (Y(II)), and (NPQ) from a typical leaf attached to $R$. meillandina plant, in control conditions, exposed to stress photoperiods $\left(18 \mathrm{~h}, 1060 \mu \mathrm{mol} . \mathrm{m}^{-2} . \mathrm{s}^{-1}\right.$ PPFD and $\left.35^{\circ} \mathrm{C}\right)$ and after one recovery photoperiod $\left(18 \mathrm{~h}, 30 \mu \mathrm{mol} . \mathrm{m}^{-2} \cdot \mathrm{s}^{-1}\right.$ PPFD and 24 $\left.{ }^{\circ} \mathrm{C}\right)$. Images are color coded according to the pattern ( 0 to $1 \times 100$ range) shown below the images. The histograms show the means \pm SE of parameters calculated from variable chlorophyll fluorescence measurements in six entire leaves.

the leaf; the mean Y(II), F and NPQ values were 0.737 , 0.104 and 0.263 (for C. morifolium (Fig. 1), and 0.785, 0.066 and 0.202 for $R$. meillandina, (Fig. 2). These results indicated that photochemical efficiency was high and that fluorescence emission and heat dissipation were low in control leaves. After one photoperiod of high illumination and heat, no significant differences were observed compared with the control leaf, but after three stress photoperiods images showed changes in some regions of the leaf with $\mathrm{Y}$ (II) decreasing and $\mathrm{F}$ and NPQ increasing (Figs. 1 and 2). After one recovery photoperiod in low light at $24^{\circ} \mathrm{C}, \mathrm{Y}(\mathrm{II}), \mathrm{F}$, and NPQ images returned to levels similar to those of the control plants. The histograms of whole leaves showed no significant variations after stress photoperiods or in control leaves. Fig. (3) shows Y(II) images of a typical S. wallisii leaf after exposure to three photoperiods of high illumination and heat, and the histograms with the mean values from the analyzed leaves. Three regions were considered in the leaf, namely, basal (B), central (C) and apical (A). The adverse effects of high illumination and heat were more pronounced in the base than in the centre and top of the leaf, with Y(II) decreasing to zero close to the base and remaining high in the centre and top of the leaf. This perhaps, was due to leaf curving during exposure to stress photoperiods to reduce the incident radiation, so that the leaf region most exposed to light during photoperiods was the base, which is the region considered in this study. Fig. (4) shows images of F, Y(II) and NPQ from the basal region of a typical $S$. wallisii leaf and the histograms with the mean values from the analyzed leaves. The control images present a homogeneous colour throughout the leaf; the mean Y(II), F and NPQ values were 0.746, 0.071, and 0.254 respectively, indicating that the photochemical efficiency was high and that fluorescence emission and heat dissipation were low in the control leaves. After the first and successive photoperiods with high illumination and heat, changes were observed and Y(II) decreased significantly. After one photoperiod of low light at $24{ }^{\circ} \mathrm{C}$, no recovery was observed, and the parameter images remained similar to those of the three stress photoperiods.

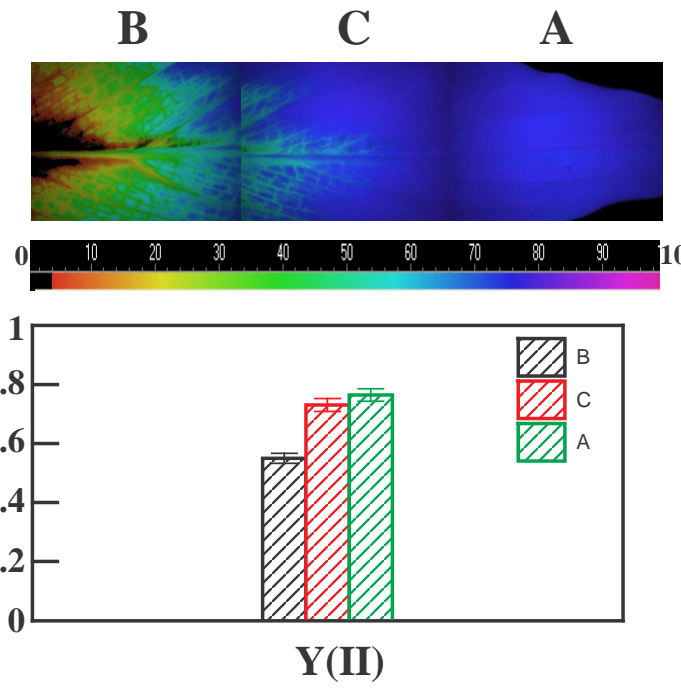

Fig. (3). Images of $(\mathrm{Y}(\mathrm{II}))$ in the apical (A), central (C) and basal (B) regions from a typical leaf attached to $S$. wallisii plant exposed to three stress photoperiods $\left(18 \mathrm{~h}, 1060 \mu \mathrm{mol} . \mathrm{m}^{-2} \cdot \mathrm{s}^{-1}\right.$ PPFD and 35 $\left.{ }^{\circ} \mathrm{C}\right)$. Images are color coded according to the pattern ( 0 to $1 \times 100$ range) shown below the images. The histogram shows the means \pm SE of the effective PS II quantum yield calculated from variable chlorophyll fluorescence measurements in six entire leaves.

\section{Effect of the Herbicides DCMU and Paraquat}

Leaves detached from sun and shade control plants were incubated with $0.1 \mathrm{mM}$ DCMU for $30 \mathrm{~min}$ and $24 \mathrm{~h}$ under white light of low intensity $\left(30 \mu \mathrm{mol} \cdot \mathrm{m}^{-2} \cdot \mathrm{s}^{-1} \mathrm{PPFD}\right)$ at $24^{\circ} \mathrm{C}$. Fig. (5) shows the images of F and Y(II) of a typical leaf of C. morifolium, R. meillandina and S. wallisii and the histograms with the mean values measured in various regions of the analyzed leaves. Control leaves incubated in water for 24 $\mathrm{h}$ showed images with a homogeneous colour throughout the leaf; the Y(II) and F mean values were 0.742 and 0.073 , respectively (for C. morifolium); 0.755 and 0.040 (for $R$. meillandina), and 0.711 and 0.070 (for $S$. wallisii), indicating that the photochemical efficiency was high and that fluorescence emission was low in the control leaves. In the three species Y(II) and F were more affected by DCMU than NPQ. After 30 min incubation with DCMU differences were observed 

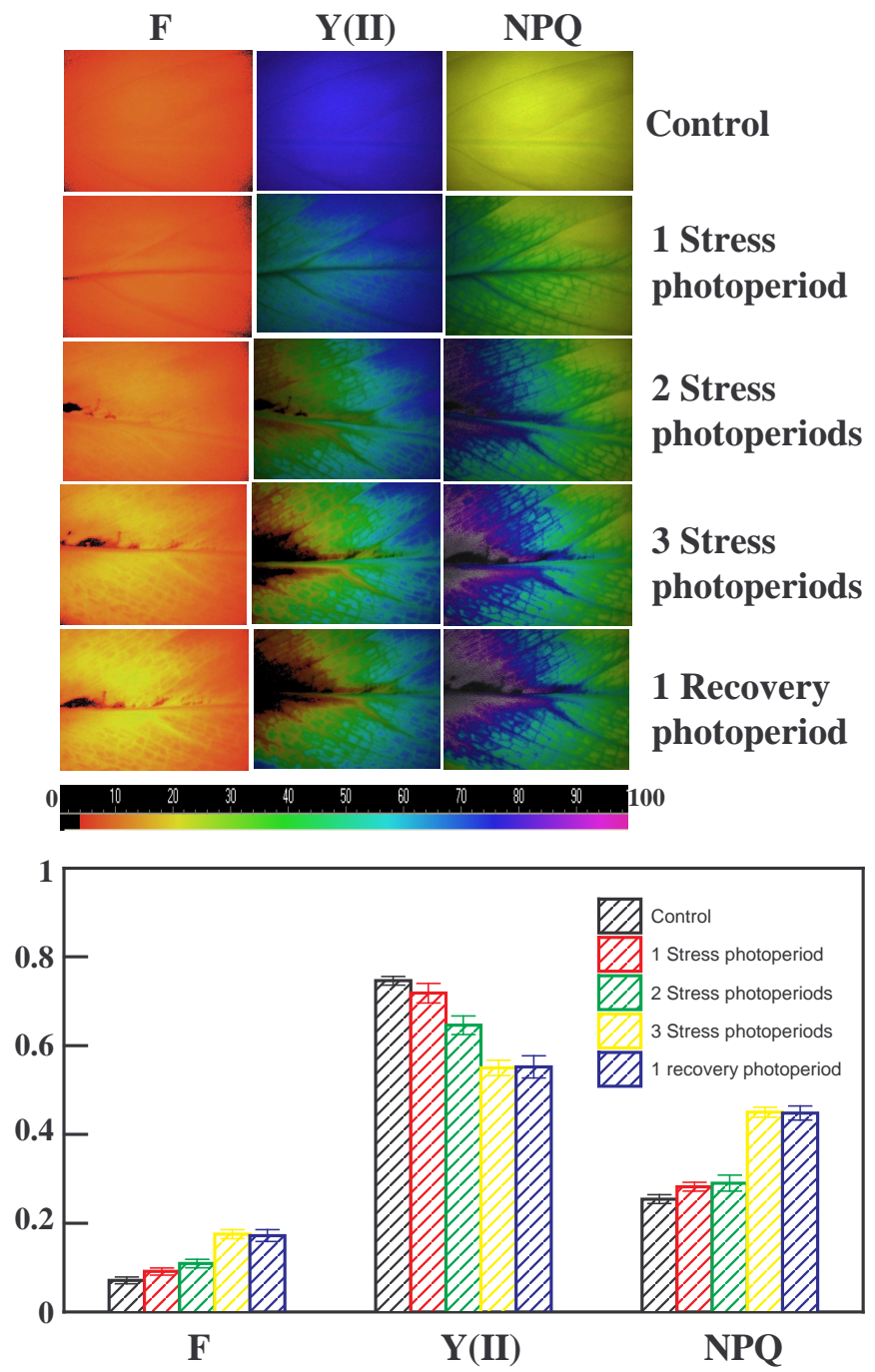

Fig. (4). Images of (F), (Y(II)), and (NPQ) from basal region of a typical leaf attached to $S$. wallisii plant, in control conditions, exposed to stress photoperiods $\left(18 \mathrm{~h}, 1060 \mu \mathrm{mol} \cdot \mathrm{m}^{-2} \cdot \mathrm{s}^{-1} \mathrm{PPFD}\right.$ and 35 $\left.{ }^{\circ} \mathrm{C}\right)$ and after one recovery photoperiod $\left(18 \mathrm{~h}, 30 \mu \mathrm{mol} \cdot \mathrm{m}^{-2} \cdot \mathrm{s}^{-1}\right.$ PPFD and $24{ }^{\circ} \mathrm{C}$ ). Images are color coded according to the pattern ( 0 to 1 $\mathrm{x} 100$ range) shown below the images. The histograms show the means \pm SE of parameters calculated from variable chlorophyll fluorescence measurements in six entire leaves.

along the main veins in all species, with Y(II) decreasing and $\mathrm{F}$ increasing in the regions near the veins. After $24 \mathrm{~h}$, the adverse effect of DCMU had spread throughout the leaf, and only at the edges of $R$. meillandina leaves did the values of $\mathrm{Y}$ (II) and $\mathrm{F}$ remained similar to those of control leaves. Fig. (6) shows the effects of incubating leaves detached from $C$. morifolium, R. meillandina and S. wallisii on $\mathrm{F}$ and $\mathrm{Y}(\mathrm{II})$. Control plants with $0.2 \mathrm{mM}$ paraquat for $30 \mathrm{~min}, 4 \mathrm{~h}, 9 \mathrm{~h}$ and $24 \mathrm{~h}$ under white light of low intensity $\left(30 \mu \mathrm{mol} \cdot \mathrm{m}^{-2} \cdot \mathrm{s}^{-1}\right.$ PPFD) at $24{ }^{\circ} \mathrm{C}$, the histograms show the mean values measured in various regions of the analyzed leaves. The control images appear similar to those from Fig. (5). In all three species Y(II) was more affected by paraquat than F and NPQ. After 30 min incubation no effect was detected in the leaves from the three species. At longer incubation times Y(II) decreased in several leaf regions and were reduced to zero in some zones. The three species showed different degrees of sensitivity to the inhibitors with the adverse effects of paraquat being clearly visible in $C$. morifolium after $4 \mathrm{~h}$ incubation, after $9 \mathrm{~h}$ in $R$. meillandina, and after $24 \mathrm{~h}$ in S. wallisii. Contrary to DCMU, the changes with paraquat did not begin in the veins but in regions distributed throughout the leaf.

\section{DISCUSSION}

Sun plants (C. morifolium and $R$. meillandina) showed greater tolerance to heat and high illumination than the shade plant (S. wallisii). In high-light conditions, the xanthophylls cycle operates, of which violaxanthin together with antheraxanthin and zeaxanthin are components [21-24]. The xanthophylls cycle is essential to prevent the rapid photoinhibition of PS II [25-27]. Sun plants accumulate zeaxanthin during high-light stress of several hours to photoprotect their photosynthetic apparatus against photoinhibition and photooxidation, whereas shade plants do not possess zeaxanthin but only its oxidized form violaxanthin with some traces of antheraxanthin, and these plants are more sensitive to photoinhibition [27].

The images presented in this paper show the differences between the leaves from sun and shade plants after stress photoperiods. In the sun plants little variations were observed in $\mathrm{F}$ and $\mathrm{Y}$ (II) images after stress photoperiods and leaf regions showed increased NPQ, indicating greater thermal energy dissipation in these zones (Figs. 1 and 2). After one recovery photoperiod, the images seemed similar to those of the control. In the shade plants visible damage was observed at the base of the leaf after stress photoperiods indicating severe photoinhibition from which there was no recovery. However, Y(II) decreased to zero in the zones adjacent to the central veins where the incident radiation was higher because the leaf curved during light exposure. For the purpose of comparison, the data for the entire leaf were also averaged and these are shown in the histograms. Integration over the entire surface of the leaf, which is equivalent to a non-imaging fluorescence measurement, did not reveal any inhibition in $C$. morifolium or $R$. meillandina after stress photoperiods, although the images showed differences between leaf regions, demonstrating the limitation of nonimaging instrumentation. In this regard, using the prompt fluorescene technique, we did not observe differences in the light response curves from control and stressed plants, under low-intensity measuring light, which conforms with previously published results [28-31]. Incubation with the herbicides DCMU and paraquat which inhibit photosynthetic electron flow, caused changes in the fluorescence parameter images. The effect of DCMU was first seen in the main veins and proximal zones, since the herbicide enters the leaf through the petiole in the vascular system [10]. After $30 \mathrm{~min}$ incubation, Y(II) decreased in the affected regions because DCMU competes with plastoquinone for the QB binding site and a large fraction of the PSII centers within these regions are driven into the closed state and the photosynthetic electron transfer is inhibited [32]. Consequently, the excitation energy is dissipated by fluorescence and the F increases considerably in the regions where photosynthesis is inhibited, blocking the energization of the thylakoid membrane and lowering non-photochemical fluorescence quenching [9]. After $24 \mathrm{~h}$ the effect of DCMU had extended throughout the leaf, especially in the shade species, since in shade plants the PSII to PSI reaction centers ratio is 3:1, compared with 2:1 in sun plants [33]. Moreover, the chloroplasts of shade plants 


\section{Chrysanthemum morifolium}

\section{Rosa meillandina}

F

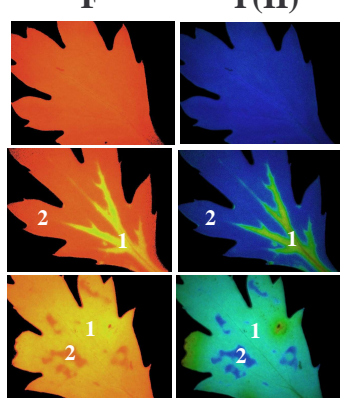

F
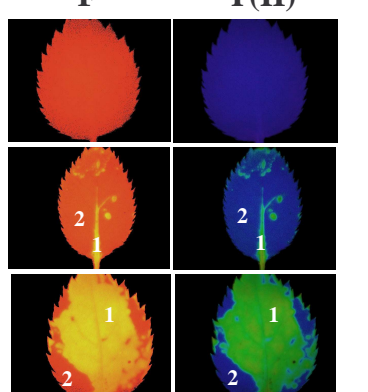

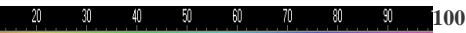
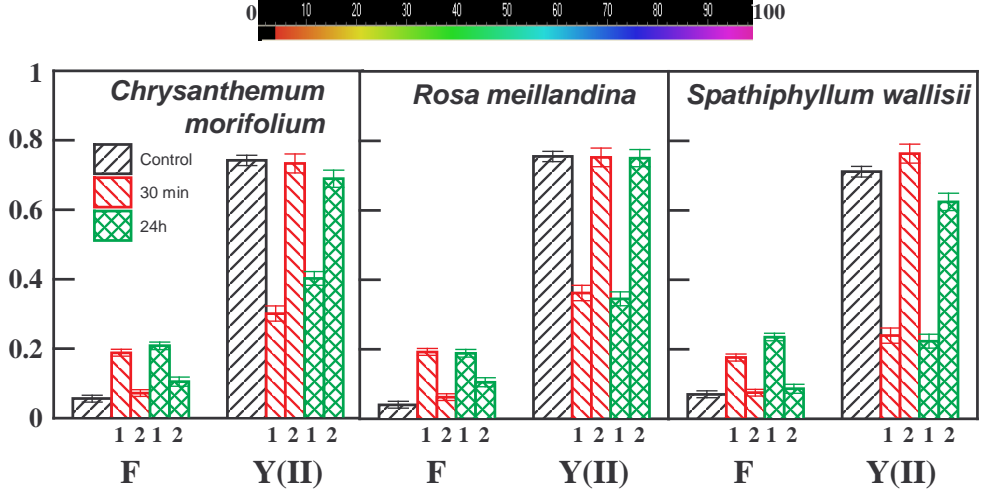

Fig. (5). Images of (F) and (Y(II)) from a typical leaf of C. morifolium, R. meillandina and $S$. wallisii incubated in $0.1 \mathrm{mM}$ DCMU for 30 min and $24 \mathrm{~h}$ under white light of low intensity $\left(30 \mu \mathrm{mol} \cdot \mathrm{m}^{-2} \cdot \mathrm{s}^{-1} \mathrm{PPFD}\right)$ at $24{ }^{\circ} \mathrm{C}$. Control was incubated in water for $24 \mathrm{~h}$. Images are color coded according to the pattern ( 0 to 1 x 100 range) shown below the images. The histograms show the means \pm SE from five different leaves of the parameters calculated from variable chlorophyll fluorescence measurements in two regions (1 and 2) of the leaf or in the entire leaf (control).
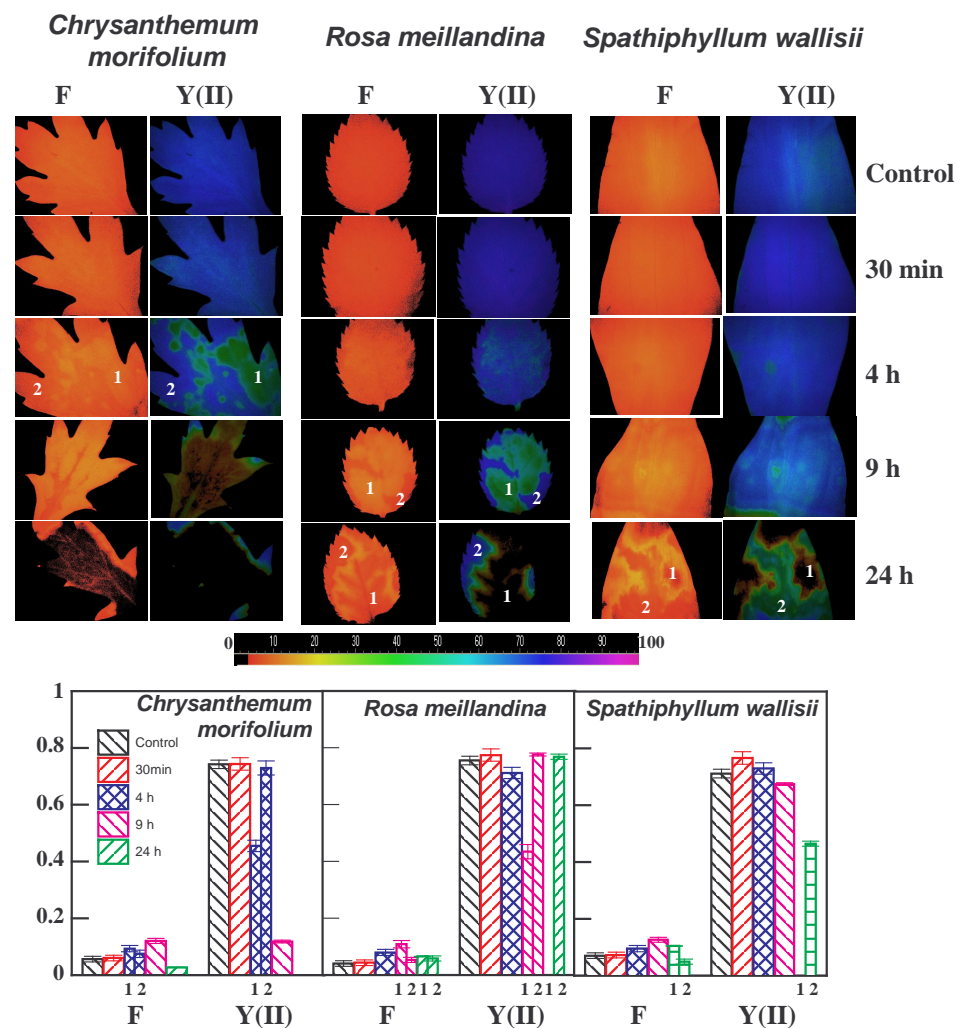

Fig. (6). Images of (F) and (Y(II)) from a typical leaf of C. morifolium, R. meillandina and S. wallisii incubated in $0.2 \mathrm{mM}$ paraquat for $30 \mathrm{~min}, 4$ $\mathrm{h}, 9 \mathrm{~h}$ and $24 \mathrm{~h}$ under white light of low intensity $\left(30 \mu \mathrm{mol} \cdot \mathrm{m}^{-2} \cdot \mathrm{s}^{-1} \mathrm{PPFD}\right)$ at $24^{\circ} \mathrm{C}$. Control was incubated in water for $24 \mathrm{~h}$. Images are color coded according to the pattern ( 0 to 1 x 100 range) shown below the images. The histograms show the means \pm SE from five different leaves of the parameters calculated from variable chlorophyll fluorescence measurements in two regions (1 and 2) of the leaf or in the entire leaf. 
possess a considerably larger antenna and the ratio of the number of chlorophyll molecules to the number of quinone molecules was about twice that in the sun plants [34]. The penetration of paraquat, a very effective auto-oxidizable electron acceptor from PS I, into leaves resulted in a gradual decrease in the PS II operating efficiency. This decrease in PS II efficiency can be attributed to the accumulation of damaged PS II complexes as the result of the rapid generation of reactive oxygen species in the presence of paraquat [35]. The three species studied showed different degrees of sensitivity to paraquat, and the effective quantum yield in each species was affected at different incubation times. All three species possessed regions in the leaves where the effective quantum yield was apparently not affected, at least at short incubation times. Similar results were also described in tobacco leaves [36]. Finally, photochemical efficiency fell to zero, which can be attributed to extensive oxidative damage in the thylakoid membranes. This variety of effects of paraquat are perhaps due to heterogeneity in its penetration, but can also indicate leaf regions with a particularly high detoxification capacity that may differ in different species, and that merits further investigation. We conclude that fluorescence imaging provides valuable information on the way in which the detrimental effects on photosynthetic activity spread through leaves and, hence, may be considered as a non-invasive tool for investigating photosynthetic activity in attached leaves when plants are subjected to adverse factors.

\section{ACKNOWLEDGEMENTS}

This work was supported by the Spanish Ministry of Education and Science (grant BFU2005-09243-C02-01).

\section{ABBREVIATIONS}

$\begin{array}{ll}\text { DCMU } & =3 \text {-(3,4-dichlorophenyl)-1,1-dimethylurea } \\ \mathrm{F} & =\text { Fluorescence yield } \\ \mathrm{Fm} & =\begin{array}{l}\text { Maximal fluorescence yield in the dark } \\ \text { adapted state }\end{array} \\ \text { F0 } & =\begin{array}{l}\text { Minimal fluorescence yield in the dark } \\ \text { adapted state }\end{array} \\ \text { F'm } & =\begin{array}{l}\text { Maximal fluorescence yield in the light } \\ \text { adapted state }\end{array} \\ \text { LED } & =\text { Light-emitting diode } \\ \text { NPQ } & =\text { Non-photochemical quenching } \\ \text { LHC } & =\text { Light harvesting complex } \\ \text { PAM } & =\text { Pulse amplitude modulation } \\ \text { Paraquat } & =N, N^{\prime} \text {-dimethyl-4, 4'-bipyridinium dichlo- } \\ \text { PPFD } & =\text { Photosynthetic photon flux density } \\ \text { PS } & =\text { Photosystem } \\ \text { Y(II) } & =\text { Effective PS II quantum yield }\end{array}$

\section{REFERENCES}

[1] Long SP, Humphries S, Falkowski PG. Photoinhibition of photosynthesis in nature. Ann Rev Plant Physiol Plant Mol Biol 1994; 45: 633-62.

[2] Papageorgiou G, Govindjee. Chlorophyll a fluorescence. A signature of photosynthesis. Springer; Dordrecht, The Netherlands 2004.
[3] Sayed OH. Chlorophyll fluorescence as a tool in cereal crop research. Photosynthetica 2003; 41;321-30.

[4] Schreiber U. Pulse-Amplitude (PAM) fluorometry and saturation pulse method. In: Papageorgiou G, Govindjee, Eds. Chlorophyll a fluorescence. A signature of photosynthesis, Springer; Dordrecht, The Netherlands 2004; pp. 279-319.

[5] Müller P, Li XP, Niyogi KK. Non-photochemical quenching: a response to excess light energy. Plant Physiol 2001; 125: 1558-66.

[6] Krause GH. Photoinhibition of photosynthesis. An evaluation of damaging and protective mechanisms. Physiol Plant 1988; 74: 56674.

[7] Krause GH, Weis E. Chlorophyll fluorescence and photosynthesis. The basics. Annu Rev Plant Physiol Plant Mol Biol 1991; 42: 31349.

[8] Allen JF. Protein phosphorylation in regulation of photosynthesis. Biochim Biophys Acta 1992; 1098: 275-335.

[9] Nedbal L, Whitmarsh J. Chlorophyll fluorescence imaging of leaves and fruits. In: Papageorgiou G, Govindjee, Eds. Chlorophyll a fluorescence. A signature of photosynthesis, Springer; Dordrecht, The Netherlands: 2004; pp. 389-407.

[10] Oxborough K. Using chlorophyll a fluorescence imaging to monitor photosynthetic performance. In: Papageorgiou G, Govindjee, Eds. Chlorophyll a fluorescence. A signature of photosynthesis, Springer; Dordrecht, The Netherlands 2004; pp. 409-28.

[11] Levitt J. Responses of plants to environmental stresses. $2^{\text {nd }}$ ed. Academic Press New York 1980; vol. 1.

[12] Bray EA, Bailey-Serres J, Weretilnyk E. Responses to abiotic stresses. In: Buchanan W, Gruissem W, Jones R, Eds. Biochemistry and Molecular Biology of Plants. American Society of Plant Physiologists; Rockville, MD 2000; pp.1158-203.

[13] Osmond CB. What is photoinhibition? Some insights from comparisons of shade and sun plants. In: Baker NR, Bowyer JR, Eds. Photoinhibition of photosynthesis: from molecular mechanisms to the field. BIOS Scientific, Oxford; United Kingdom 1994; pp.1-24.

[14] Wentworth M, Murchie EH, Gray JE, Villegas D, Pastenes C. Differential adaptation of two varieties of common bean to abiotic stress. J Exp Bot 2006; 57: 699-709.

[15] Trebst A. The topology of the plastoquinone and herbicide binding peptides of photosystem II in the thylakoid membrane. Z Naturforsch Teil 1986; C: 240-245.

[16] Crofts AR, Baroli I, Kramer D, Taoka S. Kinetics of electron transfer between QA and QB in wild type and herbicide-resistant mutants of Chlamydomonas reinhardtii. Z Naturforsch 1993; 48: 25966.

[17] Babbs CF, Pham JA, Coolbaugh RC. Lethal Hydroxyl radical production in paraquat-treated plants. Plant Physiol 1989; 90: 1267-70.

[18] Hiyama T, Ohinata A, Kobayashi S. Paraquat (methylviologen)-its interference with primary photochemical reactions. Z Naturforsch 1993; 48: 374-8.

[19] Casano LM, Martín M, Zapata JM, Sabater B. Leaf age and paraquat dependent effects on the levels of encimes protecting against photooxidative stress. Plant Sci 1999; 149: 13-22.

[20] Asada K. Production and action of active oxygen species in photosynthetic tissues. In: Foyer ChF, Mullineaux PM, Eds. Causes of photooxidative stress and amelioration of defence systems in plants. CRC Press; Boca Raton, FL 1994; pp. 77-104.

[21] Lichtenthaler HK, Burkart S, Schindler C, Stober F. Changes in photosynthetic pigments and in vivo chlorophyll fluorescence parameters under photoinhibitory growth conditions. Photosynthetica 1992; 27: 343-53.

[22] Demmig-Adams B, Adams WW. Photoprotection and other responses of plants to high light stress. Annu Rev Plant Physiol Plant Mol Biol 1993; 43: 599-626.

[23] Schindler C, Lichtenthaler HK. Is there a correlation between lightinduced zeaxanthin accumulation and quenching of variable chlorophyll a fluorescence? Plant Physiol Biochem 1994; 32: 813-23.

[24] Schindler C, Lichtenthaler HK. Photosynthetic CO2 assimilation, chlorophyll fluorescence and zeaxanthin accumulation in fieldgrown maple trees in the course of a sunny and cloudy day. J Plant Physiol 1996; 148: 399-412.

[25] Ruban AV, Horton P. Regulation of non-photochemical quenching of chlorophyll fluorescence in plants. Aus J Plant Physiol 1995; 22: 221-30.

[26] Havaux M, Gruszecki WI. Heat and light-induced Chl a fluorescence changes in potato leaves containing high or low levels of the 
carotenoid zeaxanthin, indicators of a regulatory effect of zeaxanthin on thylakoid membrane fluidity. Photochem Photobiol 1993; 58: 607-14.

[27] Lichtenthaler HK, Babani F. Light adaptation and senescence of the photosynthetic apparatus. Changes in pigment composition, chlorophyll fluorescence parameters and photosynthetic activity. In: Papageorgiou G, Govindjee, Eds. Chlorophyll a fluorescence. A signature of photosynthesis. Springer; Dordrecht, The Netherlands 2004; pp. 713-36.

[28] Quiles MJ, López NI. Photoinhibition of photosystems I and II induced by exposure to high light intensity during oat plant growth. Effects on the chloroplast NADH dehydrogenase complex. Plant Sci 2004; 166: 815-23.

[29] Quiles MJ. Stimulation of chlororespiration by heat and high light intensity in oat plants. Plant Cell Environ 2006; 29: 1463-70.

[30] Tallón C, Quiles MJ. Acclimation to heat and high light intensity during the development of oat leaves increases the NADH DH complex and PTOX levels in chloroplasts. Plant Sci 2007; 173: 438-45.
[31] Díaz M, De Haro V, Muñoz R, Quiles MJ. Chlororespiration is involved in the adaptation of Brassica plants to heat and high light intensity. Plant Cell Environ 2007; 30: 1578-85.

[32] Velthuys B. Electron dependent competition between plastoquinone and inhibitors for binding to Photosystem II. FEBS Lett 1981; 126: $277-81$

[33] Anderson JM. Photoregulation of the composition, function, and structure of thylakoid membranes. Annu Rev Plant Physiol 1986; 37: 93-136.

[34] Fork DC, Govindjee. Chlorophyll a fluorescence transients of leaves from sun and shade plants. Naturwissenschaften 1980; 67: 510-1.

[35] Váradi G, Darko E, Lehoczki E. Changes in the xanthophylls cycle and fluorescence quenching indicate light-dependent early events in the action of paraquat and the mechanism of resistance to paraquat in Erigeron canadiensis (L.). Plant Physiol 2000; 123: 1459-69

[36] Hideg E, Schreiber U. Parallel assessment of ROS formation and photosynthesis in leaves by fluorescence imaging. Photosynth Res 2007; 92: 103-8.

(C) Saura and Quiles; Licensee Bentham Open.

This is an open access article licensed under the terms of the Creative Commons Attribution Non-Commercial License (http://creativecommons.org/licenses/by$\mathrm{nc} / 3.0 /$ ) which permits unrestricted, non-commercial use, distribution and reproduction in any medium, provided the work is properly cited. 